International Journal of Pure and Applied Mathematics

Volume 85 No. 4 2013, 729-744

ISSN: 1311-8080 (printed version); ISSN: 1314-3395 (on-line version)

url: http://www.ijpam.eu

doi: http://dx.doi.org/10.12732/ijpam.v85i4.9

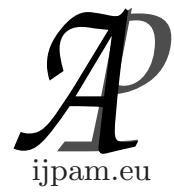

\title{
ON THE APPLICATION OF NATURAL TRANSFORMS
}

\author{
S.K.Q. Al-Omari \\ Department of Applied Sciences \\ Faculty of Engineering Technology \\ Al-Balqa Applied University \\ Amman, 11134, JORDAN
}

\begin{abstract}
In many papers, integral transforms have been introduced and found their application in solving certain boundary value problems. Indeed, as reference [1] shows, the natural transform is closely connected with Laplace and Sumudu transforms. In this paper we employ this transform to solving initial value problems of constant coefficients. Further, we discuss this transform on spaces of generalized functions. The distributional face of the natural transform is obtained as an analytic distribution. Whereas, the natural transform of a Boehmian is introduced by its limit in the distributional sense. More results are also established.
\end{abstract}

AMS Subject Classification: 54C40, 14E20, 46E25, 20C20

Key Words: natural transforms, differential equations, Sumudu transforms, Laplace transforms

\section{Introduction}

In Reference [1], a new integral transform, namely the natural transform, defined for functions of exponential order, is proclaimed.

Over the set A of functions, defined by

$$
\mathbf{A}=\left\{\begin{array}{c}
f(t) \mid \exists \mathbb{M}, \tau_{1}, \text { and } / \text { or } \tau_{2}>0, \text { such that } \\
|f(t)|<\mathbb{M} e^{\frac{|t|}{\tau_{j}}}, \text { if } t \in(-1)^{j} \times[0, \infty), j=1,2
\end{array}\right.
$$

the natural transform is defined by

Received: February 8, 2013

(c) 2013 Academic Publications, Ltd. url: www.acadpubl.eu 


$$
\mathrm{N}_{f}^{+}(u, v)=\int_{0}^{\infty} f(u t) \exp (-v t) d t, \operatorname{Re} v>0, u \in\left(-\tau_{1}, \tau_{2}\right) .
$$

where the variables $u$ and $v$ are the natural transform variables which indeed strictly converge to the Sumudu transform $[3,18]$

$$
\mathrm{M}_{f}^{+}(u)=\left\{\begin{array}{l}
\int_{0}^{\infty} f(u t) \exp (-t) d t, 0 \leq u<\tau_{2}, \\
\int_{0}^{\infty} f(u t) \exp (-t) d t,-\tau_{1}<u \leq 0,
\end{array}\right.
$$

when $v \equiv 1$ and, to the Laplace transform [10]

$$
\mathrm{L}_{f}^{+}(v)=\int_{0}^{\infty} f(t) \exp (-v t) d t, \operatorname{Re} v>0 .
$$

when $u \equiv 1$.

In notation, this is expressed to mean

$$
\mathrm{N}_{f}^{+}(u, 1)=\mathrm{M}_{f}^{+}(u) \text { and } \mathrm{N}_{f}^{+}(1, v)=\mathrm{L}_{f}^{+}(v) .
$$

The natural-Laplace duality and the natural-Sumudu duality are given in $[4,(5)-$ (8)] as

$$
\mathrm{N}_{f}^{+}(u, v)=\frac{1}{u} \int_{0}^{\infty} f(t) \exp \left(-\frac{v t}{u}\right) d t\left(\text { i.e. } \mathrm{N}_{f}^{+}(u, v)=\frac{1}{u} \mathrm{~L}_{f}^{+}\left(\frac{v}{u}\right)\right)
$$

and

$$
\mathrm{N}_{f}^{+}(u, v)=\frac{1}{v} \int_{0}^{\infty} f\left(\frac{u t}{v}\right) \exp (-t) d t\left(\text { i.e. } \mathrm{N}_{f}^{+}(u, v)=\frac{1}{v} \mathrm{M}_{f}^{+}\left(\frac{u}{v}\right)\right),
$$

respectively.

With above results it is so natural to announce linearity of the natural transform by that given to Sumudu and Laplace transforms.

The application of $\mathrm{N}$ transform to fluid flow prblems is found in [1] and, the solution of maxwell equations by the natural transform with its relation with Bessel functions are given in [2]. In some notations, if it happens, we sometimes be more convinient to express $\mathrm{N}^{+} f(u, v)$ as $\mathrm{N}_{f}^{+}(u, v)$ or $\mathrm{N}_{f}^{+}$in next investigations. 
Some facts that the natural transform enjoys are:

(1) Change of scals of non-zero integer :

$$
\mathrm{N}_{f(a t)}^{+}(u, v)=\frac{1}{a} \mathrm{~N}_{f}^{+}\left(u, \frac{v}{a}\right), a \text { non-zero integer. }
$$

(2) The natural transform of constant $a$ :

$$
\mathrm{N}_{a}^{+}(u, v)=\frac{1}{v} .
$$

(3) The natural transform of delta functions :

$$
\mathrm{N}_{\delta}^{+}(u, v)=\frac{1}{u} .
$$

(4) The natural transform of step function $u(t)=\left\{\begin{array}{l}1, t>0 \\ 0, t \leq 0\end{array}\right.$ :

$$
\mathrm{N}_{u}^{+}(u, v):=\frac{1}{v}=\left\{\begin{array}{c}
\frac{1}{v}: \mathrm{L}_{f}^{+}(v)=\mathrm{N}_{u}^{+}(1, v),(u=1) \\
1: \mathrm{M}_{u}^{+}(u)=\mathrm{N}_{u}^{+}(u, 1),(v=1)
\end{array} .\right.
$$

(5) The natural transform of $f(t)=e^{a t}, a$ constant, is :

$$
\mathrm{N}^{+}\left(e^{a t}\right)(u, v)=\frac{1}{v-a u}=\left\{\begin{array}{l}
\frac{1}{v-a}: \mathrm{L}_{f}^{+}(v)=\mathrm{N}_{u}^{+}(1, v),(u=1), \\
\frac{1}{1-a u}: \mathrm{M}_{u}^{+}(u)=\mathrm{N}_{u}^{+}(u, 1),(v=1)
\end{array} .\right.
$$

(6) The scalling property of the natural transform can be written in two ways:

$$
\mathrm{N}_{f(k t)}^{+}(u, v)=\frac{1}{k} \mathrm{~N}_{f(t)}^{+}(k u, v) \text { and } \mathrm{N}_{f(k t)}^{+}(u, v)=\frac{1}{k} \mathrm{~N}_{f(t)}^{+}\left(u, \frac{v}{k}\right) .
$$

The inverse Natural transform is related with Bromwich contour integral as [2]

$$
\mathrm{N}^{-1}(t):=f(t):=\lim _{y \rightarrow \infty} \frac{1}{2 \pi i} \int_{r-i y}^{r+i y} \mathrm{~N}_{f}^{+}(u, v) \exp \left(\frac{v t}{u}\right) d v .
$$

We describe the Parseval-Type theorem of the natural transform of an integrable function $f$ in two ways :

(i) $\int_{0}^{\infty} f(v) \mathrm{N}_{g}^{+}(v, u) d v=\int_{0}^{\infty} \mathrm{N}_{f}^{+}(t, u) g(t) d t$ 
(ii) $\int_{0}^{\infty} f(u) \mathrm{N}_{g}^{+}(v, u) d u=\int_{0}^{\infty} \mathrm{N}_{f}^{+}(v, t) g(t) d t$

Since the natural transforms $\mathrm{N}_{f}^{+}(t, u)$ and $\mathrm{N}_{g}^{+}(v, u)$ are indeed bounded and continuous for all $u$ and $v$, the integrals in $(i)$ are uniformly bounded. Therefore Fubinitz theorem implies

$$
\begin{aligned}
\int_{0}^{\infty} f(v) \mathrm{N}_{g}^{+}(v, u) d s & =\int_{0}^{\infty} f(v) \int_{0}^{\infty} g(t) \frac{1}{u} \exp \left(-\frac{v t}{u}\right) d v d t \\
& =\int_{0}^{\infty}\left(\int_{0}^{\infty} f(v) \frac{1}{u} \exp \left(-\frac{v t}{u}\right)\right) d v g(t) d t \\
& =\int_{0}^{\infty} \mathrm{N}_{f}^{+}(t, u) g(t) d t
\end{aligned}
$$

This justifies (i). Establishing (ii) can similarly be followed.

\section{Natural Transform for Ordinary Differential Equations}

By the application of [1, Theorem 2.5, Theorem 2.6] the natural transform of first and second derivatives is given as

$$
\mathrm{N}_{f^{\prime}}^{+}(u, v)=\mathrm{N}^{+}\left(f^{\prime}\right)(u, v)=\frac{v}{u} \mathrm{~N}_{f}^{+}(u, v)-\frac{f(0)}{u} .
$$

and

$$
\mathrm{N}_{f^{\prime \prime}}^{+}(u, v)=\mathrm{N}^{+}\left(f^{\prime \prime}\right)(u, v)=\frac{v^{2}}{u^{2}} \mathrm{~N}_{f}^{+}(u, v)-\frac{v}{u^{2}} f(0)-\frac{f^{\prime}(0)}{u}
$$

from which, (13) and (14) are extended to $n$th derivatives to mean [2]

$$
\mathrm{N}_{f^{(n)}}^{+}(u, v)=\mathrm{N}^{+}\left(f^{(n)}\right)(u, v)=\frac{v^{n}}{u^{n}} \mathrm{~N}_{f}^{+}(u, v)-\sum_{k=0}^{n-1} \frac{v^{n-(k+1)}}{u^{n-k}} f^{(k)}(0) .
$$

Let us make idea more precise. For, we present solutions of some initial value problems by the natural transform. A prior step of solving differential equations by the new transform is by considering the following table [1, Appendix] which is needful in the next investigation: 


\begin{tabular}{|c|c|c|c|c|c|}
\hline S.No. & $f(t)$ & $\mathrm{N}_{f}^{+}(u, v)$ & S.No. & $f(t)$ & $\mathrm{N}_{f}^{+}(u, v)$ \\
\hline 1 & 1 & $\frac{1}{v}$ & 14 & $\frac{e^{b t} \sinh a t}{a}$ & $\frac{u}{(v-b u)^{2}-a^{2} u^{2}}$ \\
\hline 2 & $t$ & $\frac{u}{v^{2}}$ & 15 & $\frac{t \sin a t}{2 a}$ & $\frac{v u^{2}}{\left(v^{2}+u^{2} a^{2}\right)^{2}}$ \\
\hline 3 & $e^{a t}$ & $\frac{1}{v-a u}$ & 16 & $t \cos a t$ & $\frac{u\left(v^{2}-a^{2} u^{2}\right)}{\left(v^{2}+a^{2} u^{2}\right)^{2}}$ \\
\hline 4 & $\frac{1}{a} \sin a t$ & $\frac{u}{v^{2}+a^{2} u^{2}}$ & 17 & $\frac{\sin a t+a t \cos a t}{2 a}$ & $\frac{v^{2} u}{\left(v^{2}+a^{2} u^{2}\right)^{2}}$ \\
\hline 5 & $\cos a t$ & $\frac{v}{v^{2}+a^{2} u^{2}}$ & 18 & $\cos a t-\frac{1}{2} a t \sin a t$ & $\frac{v^{3}}{\left(v^{2}+a^{2} u^{2}\right)^{2}}$ \\
\hline 6 & $\cosh t$ & $\frac{v}{v^{2}-u^{2}}$ & 19 & $\frac{\sin a t-a t \cos a t}{2 a^{2}}$ & $\frac{u^{3}}{\left(v^{2}+a^{2} u^{2}\right)^{2}}$ \\
\hline 7 & $\frac{t^{n-1}}{(n-1) !}, n=1,2$ & $u^{(n-1)} v^{(n-1)}$ & 20 & $\frac{a t \cos a t-\sinh t}{2 a^{3}}$ & $\frac{u^{3}}{\left(v^{2}-a^{2} u^{2}\right)^{2}}$ \\
\hline 8 & $\frac{t^{n-1}}{\Gamma(n)}, n>0$ & $u^{(n-1)} v^{(n-1)}$ & 21 & $\frac{v \sinh a t}{2 a}$ & $\frac{v u^{2}}{\left(v^{2}-a^{2} u^{2}\right)^{2}}$ \\
\hline 9 & $\cos t$ & $\frac{v}{v^{2}+u^{2}}$ & 22 & $\frac{\sinh a t+a t \cosh a t}{2 a}$ & $\frac{v^{2} u}{\left(v^{2}-a^{2} u^{2}\right)^{2}}$ \\
\hline 10 & $\sin t$ & $\frac{u}{v^{2}+u^{2}}$ & 23 & $\cosh a t+\frac{1}{2} a t \sinh a t$ & $\frac{v^{3}}{\left(v^{2}-a^{2} u^{2}\right)^{2}}$ \\
\hline 11 & $\sinh a t$ & $\frac{a u}{v^{2}-a^{2} u^{2}}$ & 24 & $t \cosh a t$ & $\frac{u\left(v^{2}+a^{2} u^{2}\right)}{\left(v^{2}+a^{2} u^{2}\right)^{2}}$ \\
\hline 12 & $\cosh a t$ & $\frac{v}{v^{2}-a^{2} u^{2}}$ & 25 & $\frac{\left(3-a^{2} t^{2}\right) \sin a t-3 a t \cos a t}{8 a^{5}}$ & $\frac{u^{5}}{\left(v^{2}+a^{2} u^{2}\right)^{3}}$ \\
\hline 13 & $e^{b t} \cosh a t$ & $\frac{v-b u}{(v-b u)^{2}-a^{2} u^{2}}$ & 26 & $\frac{\left(3-a^{2} t^{2}\right) \sin a t+5 a t \cos a t}{8 a}$ & $\frac{v^{4} u}{\left(v^{2}+a^{2} u^{2}\right)^{3}}$ \\
\hline
\end{tabular}

Example 1. Given the initial value problem

$$
y^{\prime \prime}-y^{\prime}-2 y=0
$$

with the initial conditions $y(0)=1, y^{\prime}(0)=0$.

Solution. When applying the natural transform to both sides of (16) then using linearity of $\mathrm{N}_{f}^{+}$yields

$$
\mathrm{N}_{y^{\prime \prime}}^{+}(u, v)-\mathrm{N}_{y^{\prime}}^{+}(u, v)-2 \mathrm{~N}_{y}^{+}(u, v)=0 .
$$

Applying (13) and (14) to (17) and invoking the initial conditions, after simplification, we get

$$
\mathrm{N}_{y}^{+}(u, v)=\frac{v-u}{v^{2}-v u-2 u^{2}} .
$$

Factoring the denominator of (18) gives

$$
\mathrm{N}_{y}^{+}(u, v)=\frac{v-u}{(v+u)(v-2 u)} .
$$

Epression (19) can be written as

$$
\mathrm{N}_{y}^{+}(u, v)=\frac{(6 v-12 u)+(3 v+3 u)}{(3(v+u))(3(v-2 u))} .
$$


An equivalent formula to (20) is that

$$
\mathrm{N}_{y}^{+}(u, v)=\frac{2}{3(v+u)}+\frac{1}{3(v-2 u)} .
$$

Allowing the inverse natural transform to act on (21) leads to

$$
y(t)=\frac{2}{3} \mathrm{~N}^{-1}\left(\frac{1}{v+u}\right)+\frac{1}{3} \mathrm{~N}^{-1}\left(\frac{1}{v-2 u}\right) .
$$

So, by Entry 3 of Table 1, (22) is equal to

$$
y(t)=\frac{2}{3} e^{-t}+\frac{1}{3} e^{2 t}
$$

which is our desired solution of the ordinary differential equation.

Following example establishes a solution of initial value problem of fourth order.

Example 2. Consider the initial value problem

$$
y^{(4)}-y=0 .
$$

with the initial conditions $y(0)=0, y^{\prime}(0)=1, y^{\prime \prime}(0)=0$ and $y^{\prime \prime \prime}(0)=0$.

Solution. The natural transform when applied to (23) with the presumed initial conditions and using of (15), after computations, yield

$$
\frac{v^{4}}{u^{4}} \mathrm{~N}_{f}^{+}-\mathrm{N}_{f}^{+}=\frac{v^{2}}{u^{3}}
$$

Thus, we write

$$
\mathrm{N}_{y}^{+}(u, v)=\frac{u v^{2}}{v^{4}-u^{4}} .
$$

Once again, as above, factoring denominator of (24) gives

$$
\mathrm{N}_{y}^{+}(u, v)=\frac{u v^{2}}{\left(v^{2}-u^{2}\right)\left(v^{2}-u^{2}\right)}=\frac{u v^{2}-u^{3}+u v^{2}+u^{3}}{2\left(v^{2}-u^{2}\right)\left(v^{2}-u^{2}\right)} .
$$

Simplifications yield

$$
\mathrm{N}_{y}^{+}(u, v)=\frac{1}{2} \frac{u}{v^{2}+u^{2}}+\frac{1}{2} \frac{u}{v^{2}-u^{2}} .
$$

Therefore, employing the inverse transform for (26) then checking of Entry 4 and Entry 11 of Table 1 implies

$$
y(t)=\frac{1}{2} \sin t+\frac{1}{2} \sinh t .
$$

which is indeed our desired solution. 


\section{Distributions and Boehmians}

In mathematical analysis, distributions or generalized functions are objects that generalize functions. Distributions make it possible to differentiate functions whose derivatives do not exist in the classical sense. In particular, any locally integrable function has a distributional derivative. Distributions are widely used to formulate generlaized solutions of partical differential equations, where the classical solution may not exists. Further, distributions are also important in physics and engineering where many problems naturally lead to differntial equation whose solution or initial conditions are distributions, see $[16,10,6]$.

Later, in 1983, Boehmians are objects obtained by an abstract algebraic construction to generalized distributions [17]. The original construction was motivated by regular operators [5] . Boehmians are subclass of Mikusinski operators, that are defined as equivalence classes of convolution quotients of functions. Since Boehmians were introduced, the framework of Boehmian has be used to define a variety of spaces of generalized functions and generalized integral transforms on those spaces.

One of the most youngest generalization of functions, and more particularly of distributions, is the theory of Boehmians. The name Boehmian space is given to all objects defined by an abstract construction similar to that of field of quotients. The construction applied to function spaces yields various spaces of generalized functions.

For linear space $\mathrm{Y}$ and a subspace $\mathrm{X}$ of $\mathrm{Y}$, assume, to all pair $(f, \phi),(g, \psi)$ of elements, $f, g \in \mathrm{Y}, \phi, \psi \in \mathrm{X}$, is assigned the products $f * \phi, g * \psi$ such that the following conditions are satisfied:

(1) $\phi * \psi \in \mathrm{X}$ and $\phi * \psi=\psi * \phi$.

(2) $(f * \phi) * \psi=f *(\phi * \psi)$.

(3) $(f+g) * \phi=f * \phi+g * \phi$

(4) $k(f * \phi)=(k f) * \phi=f *(k \phi), k \in \mathrm{R}$.

Let $\Delta$ be a family of sequences from $\mathrm{X}$ such that for $f, g \in \mathrm{Y}$ then:

(5) If $\left(\epsilon_{n}\right) \in \Delta$ and $f * \epsilon_{n}=g * \epsilon_{n}, n=1,2, \ldots$, then $f=g$.

(6) $\left(\epsilon_{n}\right),\left(\tau_{n}\right) \in \Delta \Rightarrow\left(\epsilon_{n} * \tau_{n}\right) \in \Delta$.

Elements of $\Delta$ are called delta sequences.

Consider the class A of pairs of sequences defined by

$$
\mathrm{A}=\left\{\left(\left(f_{n}\right),\left(\epsilon_{n}\right)\right):\left(f_{n}\right) \subseteq \mathrm{Y}^{\mathrm{N}},\left(\epsilon_{n}\right) \in \Delta\right\},
$$


for each $n \in \mathrm{N}$. if

The pair $\left(\left(f_{n}\right),\left(\epsilon_{n}\right)\right) \in \mathrm{A}$ is said to be quotient of sequences, denoted by $\frac{f_{n}}{\epsilon_{n}}$,

$$
f_{n} * \epsilon_{m}=f_{m} * \epsilon_{n}, \forall n, m \in \mathrm{N} .
$$

Two quotients of sequences $\frac{f_{n}}{\epsilon_{n}}$ and $\frac{g_{n}}{\tau_{n}}$ are said to be equivalent, $\frac{f_{n}}{\epsilon_{n}} \sim \frac{g_{n}}{\tau_{n}}$, if

$$
f_{n} * \epsilon_{m}=g_{m} * \tau_{n}, \forall n, m \in \mathrm{N} .
$$

The relation $\sim$ is an equivalent relation on $\mathrm{A}$ and hence, splits A into equivalence classes. The equivalence class containing $\frac{f_{n}}{\epsilon_{n}}$ is denoted by $\left[\frac{f_{n}}{\epsilon_{n}}\right]$. These equivalence classes are called Boehmians and the space of all Boehmians is denoted by $\mathrm{B}(\mathrm{Y}, \mathrm{X}, \Delta, *)$.

The sum and multiplication by a scalar of two Boehmians can be defined in a natural way

$$
\left[\frac{f_{n}}{\epsilon_{n}}\right]+\left[\frac{g_{n}}{\tau_{n}}\right]=\left[\frac{f_{n} * \tau_{n}+g_{n} * \epsilon_{n}}{\epsilon_{n} * \tau_{n}}\right]
$$

and

$$
a\left[\frac{f_{n}}{\epsilon_{n}}\right]=\left[\frac{a f_{n}}{\epsilon_{n}}\right], a \text { being complex number. }
$$

The operation $*$ and differentiation are defined by $\left[\frac{f_{n}}{\epsilon_{n}}\right] *\left[\frac{g_{n}}{\tau_{n}}\right]=\left[\frac{f_{n} * g_{n}}{\epsilon_{n} * \tau_{n}}\right]$ and $\mathcal{D}^{\alpha}\left[\frac{f_{n}}{\epsilon_{n}}\right]=\left[\frac{\mathcal{D}^{\alpha} f_{n}}{\epsilon_{n}}\right]$. Many a time, $\mathrm{Y}$ is equipped with a notion of convergence. The intrinsic relationship between the notion of convergence and the product $*$ are given by:

(1) If $f_{n} \rightarrow f$ as $n \rightarrow \infty$ in $\mathrm{Y}$ and, $\phi \in \mathrm{X}$ is any fixed element, then

$$
f_{n} * \phi \rightarrow f * \phi \text { in } \mathrm{Y} \text { as } n \rightarrow \infty \text {. }
$$

(2) If $f_{n} \rightarrow f$ as $n \rightarrow \infty$ in $\mathrm{Y}$ and $\left(\epsilon_{n}\right) \in \Delta$, then $f_{n} * \epsilon_{n} \rightarrow f$ in $\mathrm{Y}$ as $n \rightarrow \infty$.

The operation $*$ is extended to $\mathrm{B}(\mathrm{Y}, \mathrm{X}, \Delta, *) \times \mathrm{X}$ by :

$\star$ If $\left[\frac{f_{n}}{\epsilon_{n}}\right] \in \mathrm{B}(\mathrm{Y}, \mathrm{X}, \Delta, *)$ and $\phi \in \mathrm{X}$, then $\left[\frac{f_{n}}{\epsilon_{n}}\right] * \phi=\left[\frac{f_{n} * \phi}{\epsilon_{n}}\right]$.

In $\mathrm{B}(\mathrm{Y}, \mathrm{X}, \Delta, *)$, two types of convergence, $\delta$ and $\Delta$ convergence, are defined as follows: 
$\star$ A sequence of Boehmians $\left(\beta_{n}\right)$ in $\mathrm{B}(\mathrm{Y}, \mathrm{X}, \Delta, *)$ is said to be $\delta$-convergent to a Boehmian $\beta$ in $\mathrm{B}(\mathrm{Y}, \mathrm{X}, \Delta, *)$, denoted by $\beta_{n} \stackrel{\delta}{\rightarrow} \beta$, if there exists a delta sequence $\left(\epsilon_{n}\right)$ such that $\left(\beta_{n} * \epsilon_{n}\right),\left(\beta * \epsilon_{n}\right) \in \mathrm{Y}, \forall k, n \in \mathrm{N}$, and

$$
\left(\beta_{n} * \epsilon_{k}\right) \rightarrow\left(\beta * \epsilon_{k}\right) \text { as } n \rightarrow \infty \text {, in } \mathrm{Y}, \text { for every } k \in \mathrm{N} .
$$

The following is equivalent for the statement of $\delta$-convergence

$\star$ The sequence $\beta_{n} \stackrel{\delta}{\rightarrow} \beta(n \rightarrow \infty)$ in $\mathrm{B}(\mathrm{Y}, \mathrm{X}, \Delta, *)$ if and only if there is $f_{n, k}, f_{k} \in \mathrm{Y}$ and $\epsilon_{k} \in \Delta$ such that $\beta_{n}=\left[\frac{f_{n, k}}{\epsilon_{k}}\right], \beta=\left[\frac{f_{k}}{\epsilon_{k}}\right]$ and for each $k \in \mathrm{N}$,

$$
f_{n, k} \rightarrow f_{k} \text { as } n \rightarrow \infty \text { in Y. }
$$

$\star$ A sequence of Boehmians $\left(\beta_{n}\right)$ in $\mathrm{B}(\mathrm{Y}, \mathrm{X}, \Delta, *)$ is said to be $\Delta$-convergent to a Boehmian $\beta$ in $\mathrm{B}(\mathrm{Y}, \mathrm{X}, \Delta, *)$, denoted by $\beta_{n} \stackrel{\Delta}{\rightarrow} \beta$, if there exists $a\left(\epsilon_{n}\right) \in \Delta$ such that $\left(\beta_{n}-\beta\right) * \epsilon_{n} \in \mathrm{Y}, \forall n \in \mathrm{N}$, and $\left(\beta_{n}-\beta\right) * \epsilon_{n} \rightarrow 0$ as $n \rightarrow \infty$ in $\mathrm{Y}$.

see $[5,7,8,9,11,12,13,14,15,17]$ for more analysis.

\section{Natural Transform to Boehmians}

Denote by $E(R)$ the space of smooth functions on $R$ [12] and $E^{\prime}(R)$ its dual (congugate) space of distributions of compact support then, we can easily check that the natural transform kernel function

$$
\frac{1}{u} \exp \left(-\frac{v t}{u}\right) \in \mathrm{E}(\mathrm{R})
$$

by which we define the distributional natural transform through the inner product

$$
\widehat{\mathrm{N}}_{f}^{+}(u, v)=\left\langle f(t), \frac{1}{u} \exp \left(-\frac{v t}{u}\right)\right\rangle,
$$

$f \in \mathrm{E}^{\prime}(\mathrm{R})$.

Analogous to the classical results that assigned to the classical transform we easily establish the distributional ones.

Theorem 3. $\widehat{\mathrm{N}}_{f}^{+}$is linear.

Theorem 4. $\widehat{\mathrm{N}}_{f}^{+}$is one to one.

Theorem 5. The distributional transform $\widehat{\mathrm{N}}_{f}^{+}$is analytic and

$$
\mathcal{D}_{u} \widehat{\mathbb{N}}_{f}^{+}(u, v)=\left\langle f(t), \mathcal{D}_{u}\left(\frac{1}{u} \exp \left(-\frac{v t}{u}\right)\right)\right\rangle
$$


and

$$
\mathcal{D}_{v} \widehat{\mathrm{N}}_{f}^{+}(u, v)=\left\langle f(t), \mathcal{D}_{v}\left(\frac{1}{u} \exp \left(-\frac{v t}{u}\right)\right)\right\rangle .
$$

Proofs of Theorem 3,4 and 5 are avoided because of their similarities with that in the literature of usual transforms.

The natural transform of the convolution product

$$
(f * g)(t)=\int_{0}^{\infty} f(x) g(t-x) d x
$$

is given by aid of the Fubnitz theorem,

$$
\begin{aligned}
\mathrm{N}_{f * g}^{+}(u, v) & =\int_{0}^{\infty}(f * g)(t)\left(\frac{1}{u} \exp \left(-\frac{v t}{u}\right)\right) d t \\
& =\int_{0}^{\infty} \int_{0}^{\infty} f(x) g(t-x) d x\left(\frac{1}{u} \exp \left(-\frac{v t}{u}\right)\right) d t \\
& =\int_{0}^{\infty} f(x) \int_{0}^{\infty} g(t-x)\left(\frac{1}{u} \exp \left(-\frac{v t}{u}\right)\right) d t d x
\end{aligned}
$$

When the substitution $t-x=y$ is used, (28) yields

$$
\mathrm{N}_{f * g}^{+}(u, v)=\int_{0}^{\infty} f(x) \int_{0}^{\infty} g(y)\left(\frac{1}{u} \exp \left(-\frac{v(x+y)}{u}\right)\right) d y d x .
$$

But since $g$ can not take negative values we get

$$
\mathrm{N}_{f * g}^{+}(u, v)=u \mathrm{~N}_{f}^{+}(u, v) \mathrm{N}_{g}^{+}(u, v) .
$$

The result in (30) can be extended to distributions by the generalized convolution [16]

$$
(f * g)(t)=\langle f(t),\langle g(x), \phi(x+t)\rangle\rangle,
$$

where $\phi \in \mathrm{E}(\mathrm{R})$ to give

$$
\widehat{\mathrm{N}}_{f * g}^{+}(u, v)=u \widehat{\mathrm{N}}_{f}^{+}(u, v) \widehat{\mathrm{N}}_{g}^{+}(u, v),
$$

where $f, g \in \mathrm{E}^{\prime}(\mathrm{R})$. 
The formula (32) is sometimes written as

$$
\widehat{\mathrm{N}}_{f * g}^{+}=u \widehat{\mathrm{N}}_{f}^{+} \widehat{\mathrm{N}}_{g}^{+} .
$$

Let us consider a space of Boehmians $B(E, D, \Delta, *)$ with a group $E(R), D(R)$, the Schwartz space of test functions, as a subgroup of $E(R)(D(R)$ infact dense in $\mathrm{E}(\mathrm{R}))$ and, $*$ as an operation for $\mathrm{E}(\mathrm{R})$ and $\mathrm{D}(\mathrm{R})$ and, $\Delta$ as the set of delta sequence defined by $[4,(3.3),(3.5) \cdot(3.6)]$. Also we denote by $D^{\prime}(R)$ the strong dual of $D(R)$ of Schwartz distributions.

Theorem 6. Let $\left[\frac{f_{n}}{\psi_{n}}\right] \in \mathrm{B}(\mathrm{E}, \mathrm{D}, \Delta, *)$ then the sequence $\mathrm{N}_{f_{n}}^{+}$converges in $\mathrm{D}^{\prime}(\mathrm{R})$. Moreover, if $\left[\frac{f_{n}}{\psi_{n}}\right]=\left[\frac{g_{n}}{\varphi_{n}}\right]$ in the sense of $\mathrm{B}(\mathrm{E}, \mathrm{D}, \Delta, *)$ then $\mathrm{N}_{f_{n}}^{+}$and $\mathrm{N}_{g_{n}}^{+}$converge to the same limit.

Proof. For delta sequence $\left(\delta_{n}\right)$ it is needed to know that

$$
\mathrm{N}_{\delta_{n}}^{+}(u, v) \rightarrow \mathrm{N}_{\delta}^{+}(u, v) \text { as } n \rightarrow \infty .
$$

Hence, (9) gives

$$
\mathrm{N}_{\delta_{n}}^{+}(u, v) \rightarrow \frac{1}{u} \text { as } n \rightarrow \infty .
$$

Let $\varphi \in \mathrm{D}(\mathrm{R})$ be given such that $\mathrm{N}_{\psi_{k}}^{+}>0$ on the support of $\varphi, k \in \mathrm{N}$ (The set of natural numbers ). The hypothesis that $\frac{f_{n}}{\psi_{n}}$ is quotient of sequences implies

$$
f_{n} * \psi_{m}=f_{m} * \psi_{n}
$$

which then gives

$$
\mathrm{N}_{f_{n}}^{+} \mathrm{N}_{\psi_{m}}^{+}=\mathrm{N}_{f_{m}}^{+} \mathrm{N}_{\psi_{n}}^{+}, n, m \in \mathrm{N}
$$

Therefore we see that

$$
\begin{aligned}
\mathrm{N}_{f_{n}}^{+}(\varphi) & =\mathrm{N}_{f_{n}}^{+}\left(\varphi \frac{\mathrm{N}_{\psi_{k}}^{+}}{\mathrm{N}_{\psi_{k}}^{+}}\right) \\
& =\mathrm{N}_{f_{n}}^{+}\left(\frac{\mathrm{N}_{\psi_{k}}^{+}}{\mathrm{N}_{\psi_{k}}^{+}} \varphi\right) \\
& =\left(\mathrm{N}_{f_{n}}^{+} \mathrm{N}_{\psi_{k}}^{+}\right)\left(\frac{\varphi}{\mathrm{N}_{\psi_{k}}^{+}}\right) .
\end{aligned}
$$


By (35) we obtain

$$
\mathrm{N}_{f_{n}}^{+}(\varphi)=\left(\mathrm{N}_{f_{k}}^{+} \mathrm{N}_{\psi_{n}}^{+}\right)\left(\frac{\varphi}{\mathrm{N}_{\psi_{k}}^{+}}\right)=\mathrm{N}_{f_{k}}^{+}\left(\varphi \frac{\mathrm{N}_{\psi_{n}}^{+}}{\mathrm{N}_{\psi_{k}}^{+}}\right) .
$$

The fact that $\left(\frac{\varphi \mathrm{N}_{\psi_{n}}^{+}}{\mathrm{N}_{\psi_{k}}^{+}}\right) \rightarrow \frac{\varphi}{u \mathrm{~N}_{\psi_{k}}^{+}}$in $\mathrm{D}(\mathrm{R})$, the sequence $\mathrm{N}_{f_{n}}^{+}$converges in $\mathrm{D}^{\prime}(\mathrm{R})$. Next, let $\left[\frac{f_{n}}{\psi_{n}}\right]=\left[\frac{g_{n}}{\varphi_{n}}\right]$ in $\mathrm{B}(\mathrm{E}, \mathrm{D}, \Delta, *)$ and define

$$
\mathrm{h}_{n}=\left\{\begin{array}{c}
\frac{f_{n+1}}{2} * \frac{\varphi_{n+1}}{2}, n \text { is odd } \\
g_{\frac{n}{2}} * \psi_{\frac{n}{2}}, n \text { is even }
\end{array}\right.
$$

and

$$
\mathrm{I}_{n}=\left\{\begin{array}{c}
\psi_{\frac{n+1}{2}} * \varphi_{\frac{n+1}{2}}, n \text { is odd } \\
\psi_{\frac{n}{2}} * \varphi_{\frac{n}{2}}, n \text { is even }
\end{array}\right.
$$

then you get $\left[\frac{\mathrm{h}_{n}}{\mathrm{I}_{n}}\right]=\left[\frac{f_{n}}{\psi_{n}}\right]=\left[\frac{g_{n}}{\varphi_{n}}\right]$.

Therefore, $\mathrm{N}_{\mathrm{h}_{n}}^{+}$converges in $\mathrm{D}^{\prime}(\mathrm{R})$ by the first part.

Moreover,

$$
\lim _{n \rightarrow \infty} \mathrm{N}_{\mathrm{h}_{2 n-1}}^{+}(\varphi)=\lim _{n \rightarrow \infty} \mathrm{N}_{f_{n}}^{+}(\varphi) .
$$

Therefore $\left(\mathrm{N}_{\mathrm{h}_{n}}^{+}\right)_{n=1}^{\infty}$ and $\left(\mathrm{N}_{f_{n}}^{+}\right)$converge to the same limit.

Similarly, $\left(\mathrm{N}_{\mathrm{h}_{n}}^{+}\right)_{n=1}^{\infty}$ and $\left(\mathrm{N}_{g_{n}}^{+}\right)$converge to the same limit.

The proof is therefore completed.

This theorem can be expressed to mean that $\mathrm{N}^{+}$maps $\mathrm{B}(\mathrm{E}, \mathrm{D}, \Delta, *)$ into $\mathrm{D}^{\prime}(\mathrm{R})$.

By aid of Theorem 6 we define the natural transform of the Boehmian $\beta=\left[\frac{f_{n}}{\psi_{n}}\right] \in \mathrm{B}(\mathrm{E}, \mathrm{D}, \Delta, *)$ by

$$
\widetilde{\mathrm{N}}_{\beta}^{+}=\lim _{n \rightarrow \infty} \mathrm{N}_{f_{n}}^{+} .
$$

Theorem 7. The natural transform $\widetilde{\mathrm{N}}_{f_{n}}^{+}$is linear.

Proof is straightforward.

Theorem 8. The natural transform $\widetilde{\mathrm{N}}_{\beta}^{+}$is infinitely smooth. 
Proof. Let $\beta=\left[\frac{f_{n}}{\psi_{n}}\right] \in \mathrm{B}(\mathrm{E}, \mathrm{D}, \Delta, *)$ and $\mathrm{K}$ be an open bounded set on $\mathrm{R}$ then there is $m \in \mathrm{N}$ such that

$$
\mathrm{N}_{\psi_{m}}^{+}>0 \text { on } \mathrm{K}
$$

Hence, by (34),

$$
\begin{aligned}
\widetilde{\mathrm{N}}_{\beta}^{+} & =\lim _{n \rightarrow \infty} \mathrm{N}_{f_{n}}^{+} \\
& =\lim _{n \rightarrow \infty} \frac{\mathrm{N}_{f_{n}}^{+} \mathrm{N}_{\psi_{m}}^{+}}{\mathrm{N}_{\psi_{m}}^{+}} \\
& =\frac{1}{u} \lim _{n \rightarrow \infty} \frac{\mathrm{N}_{f_{n} * \psi_{m}}^{+}}{\mathrm{N}_{\psi_{m}}^{+}} \\
& =\frac{1}{u} \lim _{n \rightarrow \infty} \frac{\mathrm{N}_{f_{n} * \psi_{n}}^{+}}{\mathrm{N}_{\psi_{m}}^{+}} \\
& =\lim _{n \rightarrow \infty} \frac{\mathrm{N}_{f_{m}}^{+} \mathrm{N}_{\psi_{n}}^{+}}{\mathrm{N}_{\psi_{m}}^{+}} \\
& =\frac{\mathrm{N}_{f_{m}}^{+}}{\mathrm{N}_{\psi_{m}}^{+}} \lim _{n \rightarrow \infty} \mathrm{N}_{\psi_{n}}^{+} \\
& =\frac{\mathrm{N}_{f_{m}}^{+}}{u\left(\mathrm{~N}_{\psi_{m}}^{+}\right)} \text {on } \mathrm{K}
\end{aligned}
$$

but since $\mathrm{N}_{f_{m}}^{+}, \mathrm{N}_{\psi_{m}}^{+} \in \mathrm{E}(\mathrm{R})$ and $\mathrm{N}_{\psi_{m}}^{+}>0$ on $\mathrm{K}$ it follows that $\widetilde{\mathrm{N}}_{\beta}^{+}$is infinitely smooth function.

This completes the proof.

Theorem 9. If $\beta=\left[\frac{f_{n}}{\psi_{n}}\right] \in \mathrm{B}(\mathrm{E}, \mathrm{D}, \Delta, *)$ then

$$
\widetilde{\mathrm{N}}_{\beta}^{+} \mathrm{N}_{\psi_{m}}^{+}=\frac{\mathrm{N}_{f_{m}}^{+}}{u} \text { for } m \in \mathrm{N}
$$

Proof. Let $\varphi \in \mathrm{D}(\mathrm{R})$ then invoking (34) suggests

$$
\begin{aligned}
\widetilde{\mathrm{N}}_{\beta}^{+} \mathrm{N}_{\psi_{m}}^{+}(\varphi) & =\widetilde{\mathrm{N}}_{\beta}^{+}\left(\mathrm{N}_{\psi_{m}}^{+} \varphi\right) \\
& =\lim _{n \rightarrow \infty} \mathrm{N}_{f_{n}}^{+}\left(\mathrm{N}_{\psi_{m}}^{+} \varphi\right)
\end{aligned}
$$




$$
\begin{aligned}
& =\lim _{n \rightarrow \infty}\left(\mathrm{N}_{f_{n}}^{+} \mathrm{N}_{\psi_{m}}^{+}\right)(\varphi) \\
& =\frac{1}{u} \lim \left(\mathrm{N}_{f_{n} * \psi_{m}}^{+}\right)(\varphi) \\
& =\frac{1}{u} \lim _{n \rightarrow \infty}\left(\mathrm{N}_{f_{m} * \psi_{n}}^{+}\right)(\varphi) \\
& =\lim _{n \rightarrow \infty}\left(\mathrm{N}_{f_{m}}^{+} \mathrm{N}_{\psi_{n}}^{+}\right)(\varphi) \\
& =\mathrm{N}_{f_{m}}^{+}\left(\lim _{n \rightarrow \infty} \mathrm{N}_{\psi_{n}}^{+} \varphi\right) \\
& =\mathrm{N}_{f_{m}}^{+}\left(\frac{\varphi}{u}\right) \\
& =\left(\frac{\mathrm{N}_{f_{m}}^{+}}{u}\right)(\varphi) .
\end{aligned}
$$

Hence $\widetilde{\mathrm{N}}_{\beta}^{+} \mathrm{N}_{\psi_{m}}^{+}=\frac{\mathrm{N}_{\psi_{m}}^{+}}{u}$.

This complete the proof of the theorem.

Theorem 10. Let $\beta_{1}=\left[\frac{f_{n}}{\psi_{n}}\right], \beta_{2}=\left[\frac{g_{n}}{\varphi_{n}}\right] \in \mathrm{B}(\mathrm{E}, \mathrm{D}, \Delta, *)$ then

$$
\widetilde{\mathrm{N}}_{\beta_{1} * \beta_{2}}^{+}=u \widetilde{\mathrm{N}}_{\beta_{1}}^{+} \widetilde{\mathrm{N}}_{\beta_{2}}^{+} \text {. }
$$

Proof. of this theorem follows from (9) and (36) and, routine technique similar to that of Theorem 9. Details thus avoided.

The theorem is completely proved.

\section{References}

[1] Khan, Z. H. and Khan, W.A., Natural Transform-Properties and Applications, NUST Journal of Engineering Sciences, 1,1(2008), 127-133.

[2] R. Silambarasn and F. B. M. Belgacem, Applications of the Natural Transform to Maxwell's Equations, Progress In Electromagnetics Research Symposium Proceedings, Suzhou, China, Sept. 12, 16( 2011)

[3] Adem Kiliçman, Hassan Eltayeb, and Ravi P. Agarwal, On Sumudu Transform and System of Differential Equations, Abstr. Appl. Anal. Vol. 2010, Article ID 598702.

[4] Belgacem, F. B. M. and Silambarasan, R. , Advances in the Natural transform, AIP Conf. Proc. 1493, 106 (2012); doi: 10.1063/1.4765477. 
[5] Boehme, T.K. , The Support of Mikusinski Operators, Tran.Amer. Math. Soc., 176,(1973), 319-334.

[6] Banerji, P.K., Al-Omari, S.K.Q. and Debnath, L. Tempered Distributional Fourier Sine(Cosine)Transform, Integ. Trans. Spl. Funct.

[7] Mikusinski, P. , Fourier transform for integrable Boehmians, Rocky Mountain Journal of Mathematics, 17, 3(1987), 577-582.

[8] Mikusinski, P., Tempered Boehmians and Ultradistributions, Proc. Amer. Math. Soc. 123, 3 (1995), 813-817.

[9] Al-Omari, S.K.Q., Loonker D., Banerji P.K. and Kalla, S.L. . Fourier Sine(Cosine) Transform for Ultradistributions and their Extensions to Tempered and UltraBoehmian spaces, Integ. Trans. Spl. Funct. 19, 6(2008), $453-462$.

[10] Zemanian, A.H., Generalized Integral Transformation, Dover Publications, Inc., New York. First published by interscience publishers, New York(1987).

[11] Al-Omari, S.K.Q.. Generalized Functions for Double Sumudu Transformation, Intern.J. Algeb., 6, 3(2011), 139 - 146.

[12] S.K.Q.Al-Omari and Kilicman, A.. Note on Boehmians for Class of Optical Fresnel Wavelet Transforms, Journal of Function Spaces and Applications, Vol. 2012, Article ID 405368, doi:10.1155/2012/405368.

[13] S.K.Q.Al-Omari and Kilicman, A. On Generalized Hartley-Hilbert and Fourier-Hilbert Transforms, Advances in Difference Equations, Vol. 2012, 2012:232 doi:10.1186/1687-1847-2012-232.

[14] Al-Omari, S.K.Q.. Notes for Hartley Transforms of Generalized Functions , Italian J. Pure Appl. Math. 28(2011), 21-30.

[15] S. K. Q. Al-Omari and A. Kilicman, On Diffraction Fresnel Transforms for Boehmians, Abstract and Applied Analysis, Vol. 2011, Article ID 712746.

[16] Pathak, R. S.. Integral transforms of Generalized Functions and their Applications, Gordon and Breach Science Publishers, Australia , Canada, India, Japan(1997).

[17] Mikusinski, P.. Convergence of Boehmians, Japan. J. Math. 9(1983), 159179. 
[18] Adem Kilicman, Hassan Eltayeb and Mat Rofa Ismail, A Note on Integral Transforms and Differential Equations, Malaysian Journal of Mathematical Sciences 6(S): Special Edition of International Workshop on Mathematical Analysis (IWOMA)(2012), 1-18. 